\title{
Le sable du point de vue de la physique
}

Sand: physics' perspectives

\section{Ahmed Ould el-Moctar}

\section{(2) OpenEdition}

\section{Journals}

Édition électronique

URL : https://journals.openedition.org/tc/7198

DOI : $10.4000 /$ tc. 7198

ISSN : 1952-420X

\section{Éditeur}

Éditions de l'EHESS

\section{Édition imprimée}

Date de publication : 15 décembre 2013

Pagination : 28-41

ISBN : 978-2-7351-1654-6

ISSN : 0248-6016

\section{Référence électronique}

Ahmed Ould el-Moctar, "Le sable du point de vue de la physique », Techniques \& Culture [En ligne], 61 | 2013, mis en ligne le 15 décembre 2016, consulté le 29 septembre 2022. URL : http:// journals.openedition.org/tc/7198; DOI : https://doi.org/10.4000/tc.7198 


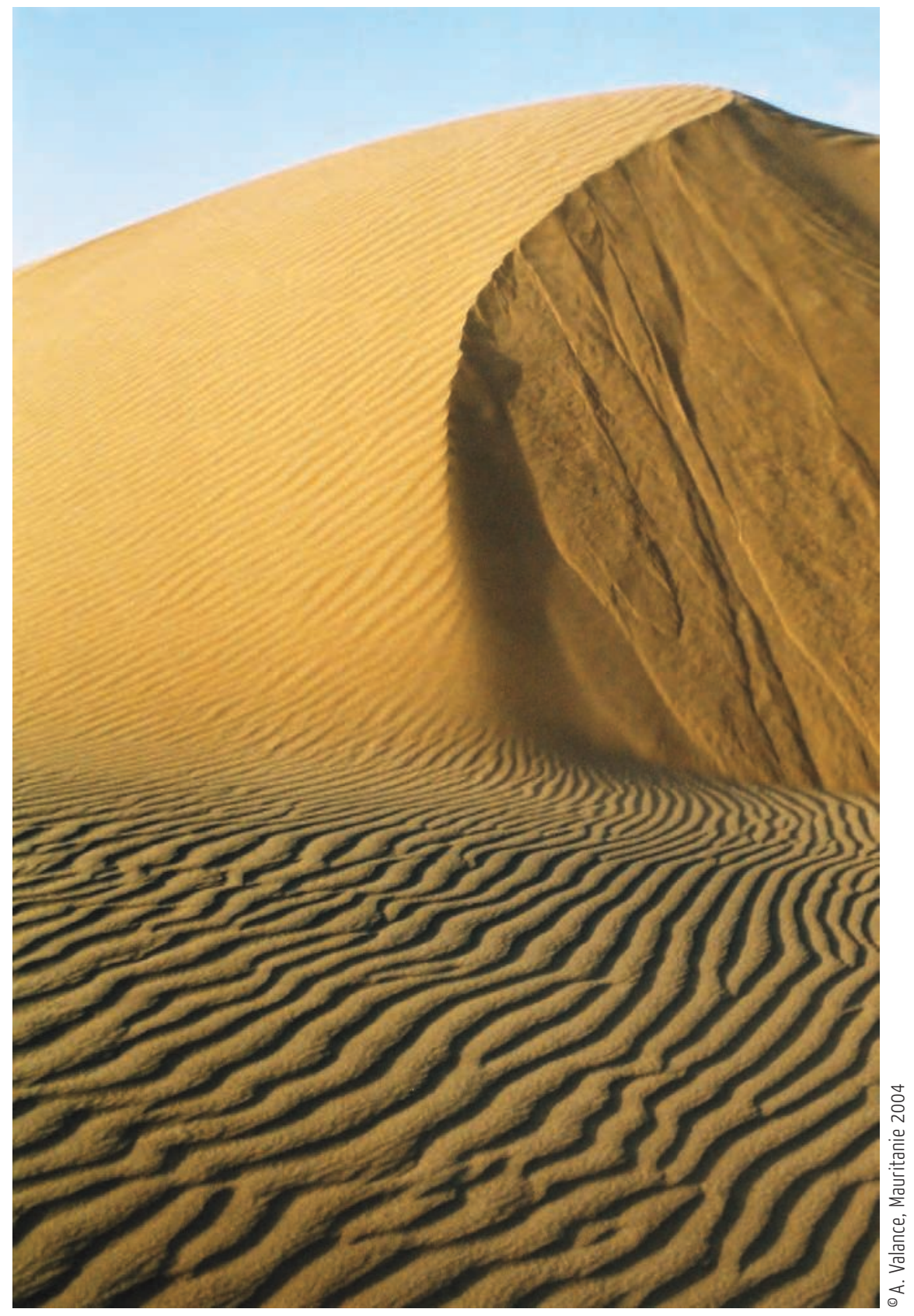


Ahmed Ould el-Moctar

Laboratoire de Thermocinétique de Nantes,

CNRS UIMR 6607

ahmed.ould-el-moctar@univ-nantes.fr
Vivre le sable! Corps, matière et sociétés - 1

Techniques \& Culture 61, 2013/2: 28-41

\section{LE SABLE}

DU POINT DE VUE DE LA PHYSIQUE

Pour tout Saharien qui a grandi entouré de sable, ce matériau peut être presque banal. On aurait pu parler de matière sable, mais s'agissant de ses propriétés, nous utiliserons plutôt le terme matériau pour désigner le sable en tant qu'objet d'étude. En effet, ce Saharien a probablement eu maintes occasions de détester le sable lors de tempêtes, par exemple, mais aussi de l'adorer par temps clément en marchant sur une dune. Le sable accompagne tous les moments de la vie, du moins dans la région saharienne dont je suis issu. Il peut servir de couche pour y naître, d'un formidable terrain de jeux, également de support à un écrit éphémère. Il peut être considéré comme sale, mais également comme propre, puisque l'on peut l'utiliser comme matière abrasive pour nettoyer les ustensiles de cuisine. Il est même possible de s'en enduire pour se purifier ou se préparer à s'acquitter d'un devoir religieux (en l'absence d'eau).

Dans tous les cas, le sable est l'un des éléments qui surgissent à l'esprit en cas de nostalgie du pays. D'ailleurs dans l'une des langues locales « hassanya », le mot trabe peut désigner tout à la fois le sable, la terre et le pays. Cette banalisation du terme est certainement due à la proximité du sable. En effet les habitants de cette région ont tous avalé un jour ou l'autre un peu de sable, et ce dernier fait partie d'eux-mêmes. Ce regard change énormément lorsque le sable devient un objet de recherche scientifique; et la fascination du chercheur pour son sujet contraste soudainement avec la banalisation du sable chez le Saharien. Les considérations sociales sont là pour vous rappeler que vouer un tel intérêt au sable relèverait aux yeux du citoyen commun d'une oisiveté certaine, voire d'une marginalité. Par contre, devant les enjeux et les problèmes posés par le sable dans les milieux arides, ce sentiment s'efface assez vite et la curiosité utile du chercheur devient légitime et compréhensible. 
Les problèmes d'ordre environnemental (désertification), économique (ensablement des routes, chemins de fer, terres cultivables) et social (ensablement d'habitations, exode rural...) qui sont liés au déplacement du sable sont tels, que les physiciens ont été nombreux à se préoccuper de cette thématique ces dernières décennies. La ville historique de Chinguetti en Mauritanie représente une bonne illustration des problèmes d'ensablement. En effet, cette ville classée patrimoine de l'humanité a été ensevelie par deux fois et chaque fois une nouvelle ville a été reconstruite à côté de l'ancienne. C'est ainsi que l'on en est rendu actuellement à la troisième ville encore menacée par l'océan de dunes de sable qui l'entoure.

Ces zones arides constituées essentiellement de déserts de sable concernent plusieurs zones du globe terrestre. Elles occupent plus de 16 millions de $\mathrm{km}^{2}$ (entre 35 et $37 \%$ de la surface émergée de la terre (Mainguet 1995) dont 8 millions de $\mathrm{km}^{2}$ pour le seul Sahara.

Répartition des déserts

Sahara, Namib et Kalahari en Afrique, Gobi et Taklamakan en Asie de l'Est, Great Sandy Simpson

en Australie, Atacama et Sierra Nevada en Amérique

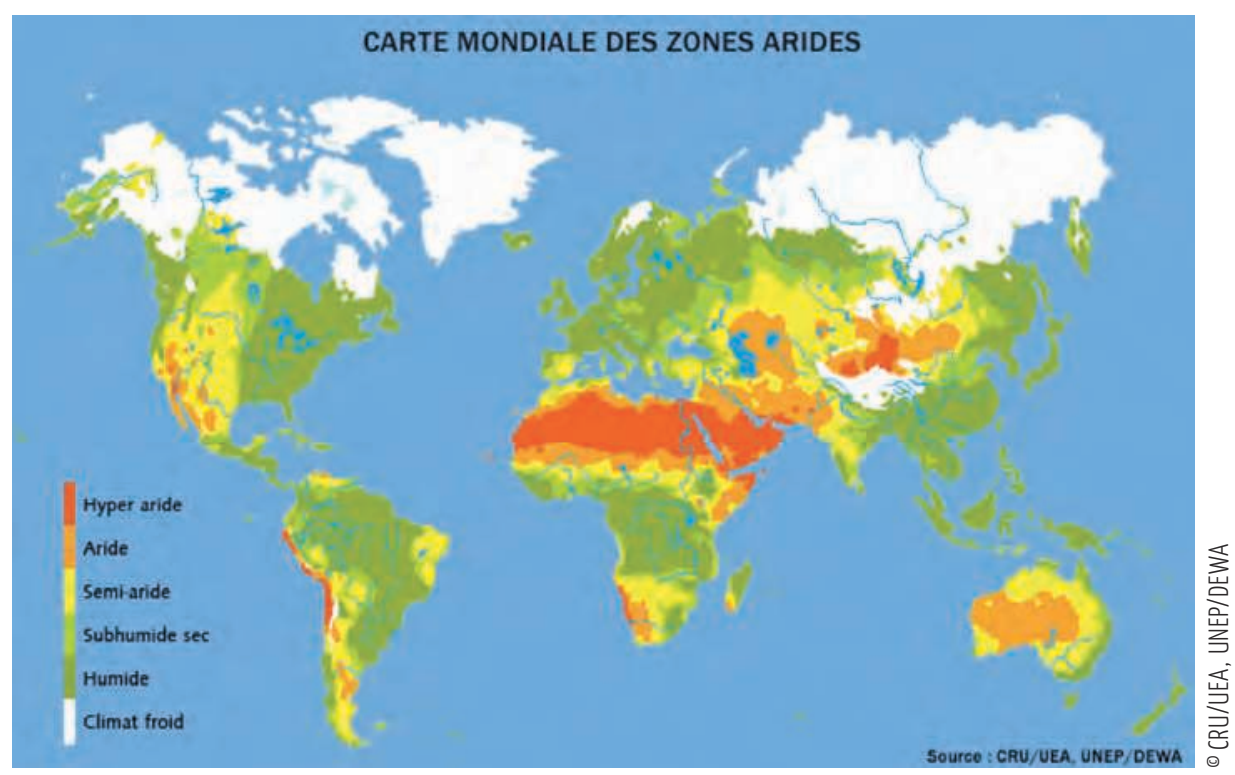

Le sable peut être considéré comme matériau constitutif d'un ensemble parmi d'autres éléments. C'est ainsi qu'il peut être mélangé avec du ciment pour constituer des matériaux de construction. Il peut être chauffé pour servir de moyen de cuisson ou mouillé pour le stabiliser ou s'en servir pour des constructions éphémères de jeux d'enfants. Dans le Sahara, après une pluie, les enfants construisent des cases en utilisant leurs pieds comme moule. Ils construisent la case au-dessus de leurs pieds et en les retirant, ils libèrent l'intérieur et la porte de la construction.

D’autre part, le sable peut interagir avec les éléments avec lesquels il est en contact tout en restant bien identifié. C'est ainsi qu'il réagit avec l'air et l'eau en état statique ou quand il est transporté mais il a aussi une action sur les éléments qu'il rencontre par l'intermédiaire de l'érosion. Cependant la manifestation la plus remarquable de l'interaction du 
sable avec son milieu est le phénomène d'ensablement quand il se dépose et entoure ou ensevelit les objets ou infrastructures qu'il rencontre sur son chemin.

Dans la suite de ce texte, nous allons présenter quelques aspects de ce matériau sable avec un regard de physicien. Il s'agira d'une présentation multi-échelle (dans le sens où les phénomènes physiques peuvent être impliqués au niveau de la taille du grain de sable, à la taille d'un amas de sable ou à celle d'un champ dunaire) plus axée sur le comportement et la dynamique permettant le transport de ce matériau et la formation d'objets présentant une morphologie particulière (les dunes).

Du point de vue du physicien, le sable entre dans la catégorie des « milieux divisés », dits «matière molle » ou des « milieux granulaires ». Dans ce sens, il partagera des caractéristiques avec par exemple des tas de grains de céréales, de billes de verre ou des cailloux de ballast.

Nous considérerons ici essentiellement le matériau granulaire sec en contact avec l'air et nous entendrons par sable, les grains de silice qui résultent naturellement de l'érosion éolienne ou pluviale des rochers plus massifs. Ces grains sont assez bien façonnés par l'érosion et présentent une forme plus ou moins sphérique. Leur granulométrie s'étale typiquement de quelques dizaines de micromètres jusqu'à l'ordre du millimètre. On peut donc dire en simplifiant qu'à l'origine, on a une roche qui par érosion donne du sable, et qui, à petite échelle, (de l'ordre du micromètre) devient poussière. La couleur rouge du sable éolien est liée aux réactions de ces grains de sable avec des oxydes de fer. Cette pellicule de rouille peut être enlevée par les impacts entre les grains de sable. C'est ainsi qu'une dune peu mobile sera constituée de sable plus rouge que celui composant une dune très mobile, où les chocs sont plus fréquents.

\section{Le sable dans tous ses états}

Le sable peut avoir un comportement allant de celui du solide à celui du gaz en passant par le liquide. En effet, si l'on verse du sable sous forme d'un tas sur une surface plane, ce tas statique aura sa forme conique bien connue avec un angle d'équilibre avoisinant les $30^{\circ}$. La surface libre n'étant pas horizontale, le sable se comporte ici comme à l'état solide. Ce sable versé dans un récipient épouse bien la surface de ce dernier ce qui est le propre de l'état liquide. Si maintenant on dispose quelques grains de sable dans un bocal et que l'on secoue, les grains ainsi agités se comporteront comme les molécules d'un gaz.

On peut imaginer la situation suivante où les trois états coexistent. Prenons une dune soumise à un fort vent, la grande masse de sable constituant la dune est globalement statique et se comporte comme un solide. Au niveau de la face d'avalanche, une fois l'angle de repos dépassé, une couche de sable s'écoule selon la pente en gardant une certaine cohésion comme un liquide. Au-dessus de la dune, dans la couche d'air chargée en particules, les grains de sable se comporteront alors comme un gaz.

Cette diversité des états possibles pour un tas de sable (du fluide au solide) est en grande partie à l'origine de la difficulté d'avoir une théorie précise pour le comportement d'un tel tas, et ceci malgré les avancées de la physique et les efforts importants de recherche dans ce domaine. 


\section{Du grain de sable au champ dunaire}

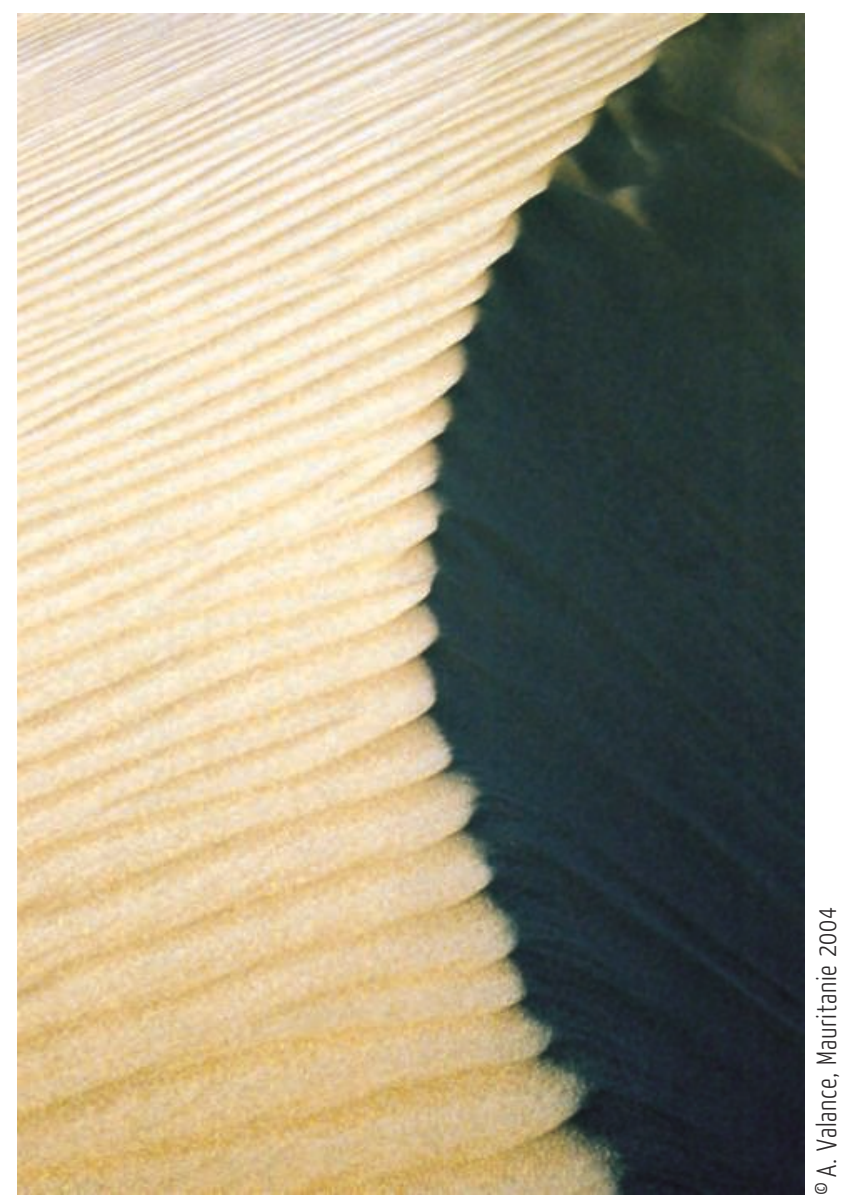

Rides éoliennes sur le sommet d'une barkhane. On distingue la ligne de crête et la face d'avalanche de la dune.
Si l'on considère un tas de sable, il est formé de grains de sable disposés de façon désordonnée (par rapport à des billes que l'on aurait arrangées de façon ordonnée). Ce désordre a pour conséquence que l'espace occupé par le fluide entre les grains (fluide interstitiel) est plus important que dans le cas ordonné. Cette organisation et les failles qui peuvent exister sont très importantes dans la physique de ce milieu spécifique. Des phénomènes tels que la distribution des contraintes, les écoulements et la ségrégation (dans le cas de particules de tailles différentes) vont en dépendre.

La forme et la présence de grains de sable de tailles différentes (polydispersité) représentent une autre difficulté dans l'étude physique de ce milieu. Ajoutons à cela d'autres paramètres comme l'humidité ou les forces électrostatiques qui peuvent influencer l'interaction entre les particules et la cohésion du milieu.

Autant dire que, même si le physicien considère en général un milieu composé par des grains sphériques et de même taille, le tas de sable, pour ne pas évoquer des structures plus complexes telles que les rides ou les dunes, reste un objet complexe à appréhender.

L'analyse de la physique des tas de sable fait ressortir des phénomènes curieux et inattendus (Claudin 1999). Ces phénomènes sont essentiellement liés à la distribution des contraintes dans le tas qui se fait selon des chaînes relatives aux contacts entre les grains et à l'équilibre de ces derniers. Cette répartition des forces, pouvant être diffractées vers les côtés, n'est donc pas verticale comme pour les autres matériaux. On parle alors d'un effet de voûte qui explique certaines situations, par exemple:

- Si l'on considère un tas posé sur un ensemble de capteurs de pression (équivalent à une pesée) on s'attend à avoir un maximum de pression au centre qui correspond à une hauteur maximale. Le résultat montre de façon surprenante qu'il y a plutôt deux maxima décalés vers les côtés et un «trou » de pression au centre. Ceci est valable pour un tas construit à partir d'un entonnoir et c'est la trajectoire des particules qui se déplacent obliquement selon des avalanches qui privilégient cette répartition des forces. Si par contre le tas est construit par couches successives la distribution de pression ne présente pas alors de minimum au centre, mais un plateau.

- Observons un tube ouvert aux deux extrémités et dont la longueur est assez grande par rapport au diamètre. Le tube est fixé et le fond est relié à une balance. Si l'on mesure, tout en remplissant peu à peu le tube de sable, la masse qui pèse sur le fond du tube, on s'attend à avoir exactement la masse versée. Ceci est effectivement le cas quand on verse une petite quantité de sable, mais très rapidement on constate que la masse qui pèse sur le fond croit moins vite 
que celle qui est versée. Cette masse mesurée au fond du tube finit par « saturer » et ne plus augmenter bien que l'on continue à verser du sable. On parle alors de « masse apparente ». Ainsi donc, à cause de cet effet de voûte, les parois du tube ont supporté une partie du poids. C'est ce qui explique aussi que les parois d'un silo à grains doivent être conçues pour supporter des forces très importantes.

- Ce phénomène de voûte éclaire également l'écoulement du sable à travers un petit orifice dans un cône comme peut l'illustrer un sablier. Effectivement, dans ce cas, le sable s'écoule de façon régulière, et le débit n'est pas influencé par la hauteur du sable. Autrement dit, on peut s'attendre à ce que plus le niveau du sable est haut plus la pression sur l'orifice est forte, et plus l'écoulement de sable est rapide. En fait il n'en est rien, à partir d'une certaine hauteur ce sont les parois latérales qui supportent le poids, et l'orifice subit toujours la même pression.

Un autre phénomène présent dans le comportement de ces milieux est celui de la ségrégation. Lorsqu'on dispose des grains de sable de différentes tailles dans un récipient que l'on secoue, on retrouve de façon inattendue les gros grains à la surface et les petits au fond. Ceci se justifie par le fait que lors des vibrations, des espaces se libèrent et sont occupés par les petites particules qui soulèvent les grosses; et ce phénomène est bien démontré par les femmes quand elles roulent les grains de semoule. Pour obtenir des grains de taille uniforme, elles enduisent les petits grains mouillés de farine et isolent les gros grumeaux en imposant une vibration de l'ensemble avec une petite inclinaison du plateau. Les gros grains ainsi isolés par ségrégation sont cassés pour en faire des plus petits et ainsi de suite.

Dans la nature généralement, et dans le désert en particulier, le sable ne s'organise pas seulement en forme de tas ou du moins les tas ou les édifices éoliens ont des morphologies bien précises. En les énumérant par ordre de taille croissant on peut distinguer :

— les rides qui présentent des longueurs caractéristiques de l'ordre de quelques centimètres. Elles se forment d'ordinaire sur les dunes et s'organisent selon la direction perpendiculaire au vent dominant. Basons-nous sur le schéma pour donner l'explication de Bagnold (1941) concernant la formation de ces rides. Une surface plane constituée de sable est forcément instable compte tenu de la répartition initiale des grains de sable et de leur réaction au vent. Cette instabilité donne lieu à une déformation de la surface qui est accentuée par le bombardement de celle-ci par les particules transportées par le vent. Si l'on examine cette petite déformation initiale, sur la portion $\mathrm{BC}$, le nombre d'impacts est supérieur à celui en $\mathrm{AB}$. Les grains heurtés en $\mathrm{BC}$ tendent donc à s'accumuler en $C$; la hauteur du point $C$ augmente et le versant $C D$ est alors de moins en moins exposé, ce qui accentue le creux en D. La formation des rides est ainsi initiée. Il existe d'autres modèles, mais pas vraiment de certitude sur le mécanisme de formation et la sélection de la longueur d'ondes caractérisant ces rides.

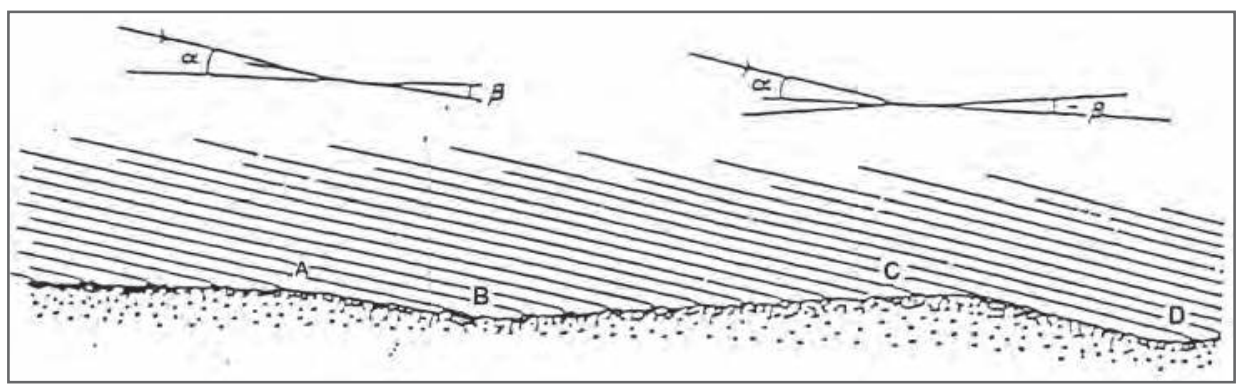

\section{Figure 3}

Disparité des impacts sur une surface légèrement déformée : initiation des rides. Bagnold (1941) 


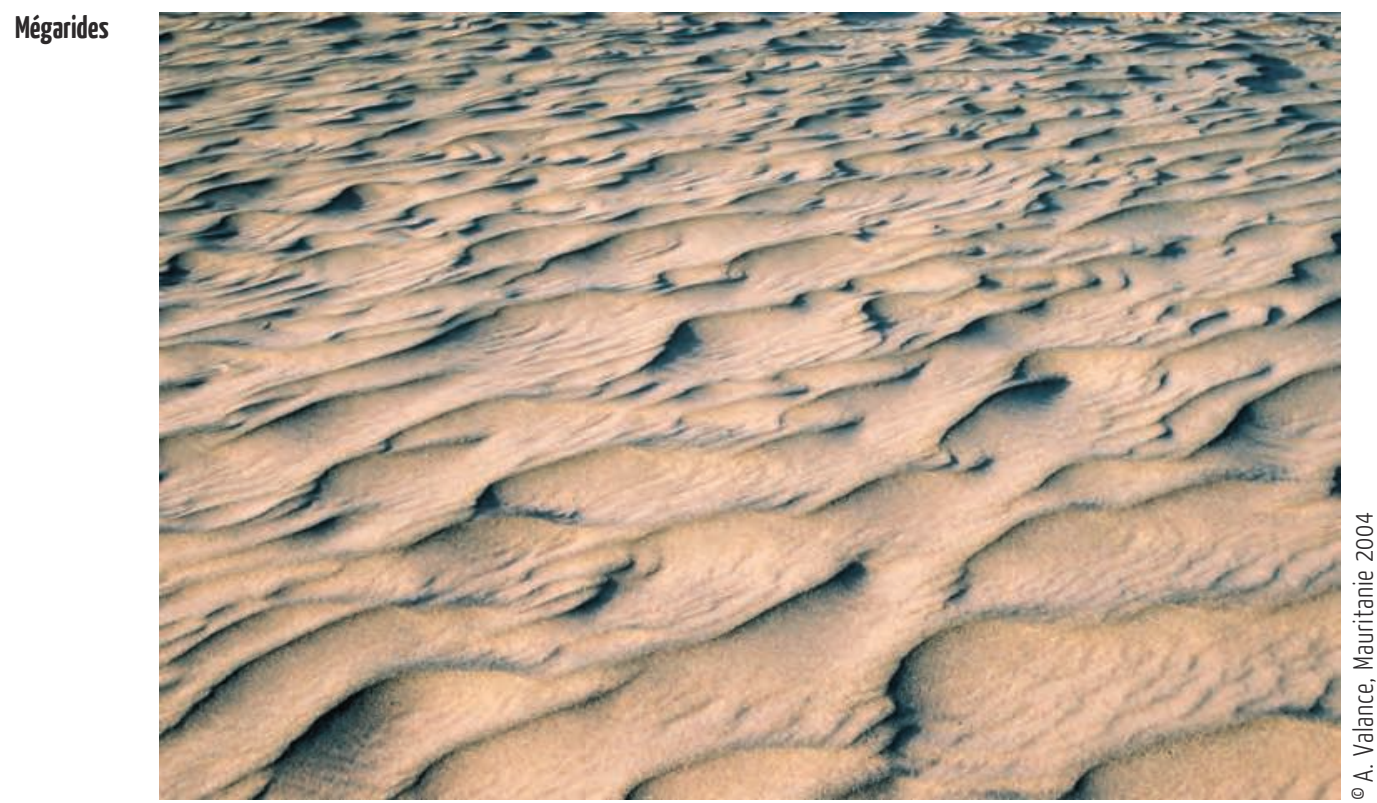

En présence d'une variation importante de la granulométrie du sable allant jusqu'à des grains de grande taille (supérieur au $\mathrm{mm}$ ), on assiste à la formation de rides de plus grande taille que l'on peut qualifier de mégarides. Ces rides présentent des longueurs caractéristiques de l'ordre du mètre. En effet, les particules les plus grosses se trouvent toujours sur les sommets ou crêtes des rides, protégeant ainsi les particules plus fines qui se trouvent dans les creux. La figure placée en ouverture d'article présente la cohabitation de ces deux tailles de rides. Les petites rides sur la dune et les grosses rides

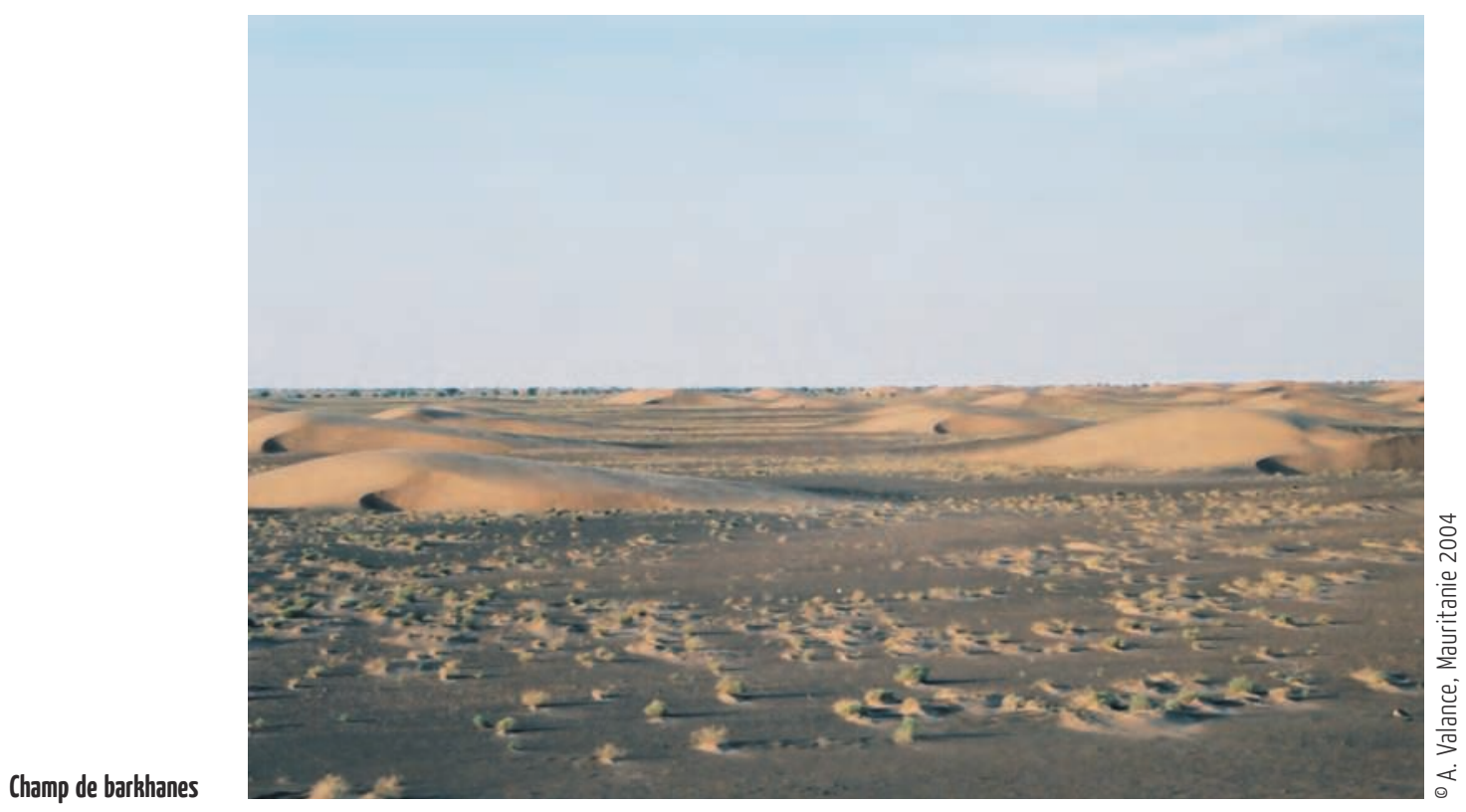


avec des particules plus grosses en bas de la dune. On peut y voir aussi une avalanche qui vient de se déclencher.

— Les dunes représentent de plus grands édifices avec des longueurs caractéristiques de l'ordre de quelques dizaines de mètres pour les barkhanes et plus pour les autres formes de dunes. La barkhane est la forme la plus simple des dunes. Elle se présente sous forme d'un croissant et peut se déplacer de plusieurs dizaines de mètres en moyenne sur une année. En fonction de l'état du sol, de la source de sable disponible et du régime des vents on observera différentes morphologies des dunes de sable. Comme indiqué dans le tableau, on peut distinguer les barkhanes, les dunes transversales, les dunes longitudinales et celles sous forme d'étoile.

\begin{tabular}{||l|l|l||}
\hline \multicolumn{1}{|c|}{ Types } & \multicolumn{1}{|c|}{ Conditions } \\
\hline \hline \multirow{5}{*}{ Barkhane } & $\begin{array}{l}\text { - Surfaces rocailleuses } \\
\text { - Approvisionnement } \\
\text { en sable modéré } \\
\text { - Direction du vent } \\
\text { constante }\end{array}$ \\
\hline Dunes transverses & $\begin{array}{l}\text { - Approvisionnement } \\
\text { en sable abondant } \\
\text { - Direction du vent } \\
\text { constante }\end{array}$ & \\
\hline Dunes longitudinales & $\begin{array}{l}\text { - Approvisionnement } \\
\text { en sable limité } \\
\text { - Au moins } 2 \text { directions } \\
\text { de vent convergentes }\end{array}$ & \\
\hline Dunes étoiles & $\begin{array}{l}\text { - Rectilignes et longues } \\
\text { en sable abondant } \\
\text { de vent }\end{array}$ & \\
\hline & & \\
\hline
\end{tabular}

Une façon d'opérer une classification de ces structures consisterait à considérer leur taille, leur forme, ainsi que le gisement de sable disponible et le régime des vents. Cette classification dite naturaliste (Mainguet 1995) n'est pas forcément la plus pertinente du point de vue de la physique. Une classification physique se baserait plutôt sur les mécanismes physiques (cisaillement, instabilité...) mis en jeu dans la formation de ces édifices de sable (Andreotti et al. 2011). Ces méthodes, naturaliste et physique, de classification ne sont pas compatibles. Ainsi, dans la méthode dite naturaliste, si l'on s'en tient uniquement à la taille, on trouvera les rides sous-marines et les rides éoliennes de l'ordre de quelques dizaines de centimètres. Dans un ordre un peu plus grand, les dunes marines et les méga-rides de l'ordre du mètre, les dunes éoliennes de l'ordre de quelques dizaines de mètres et dans un autre, bien plus grand, les dunes géantes éoliennes sur Terre et comme on le verra plus loin sur Vénus ou sur Mars. Ces dernières peuvent dépasser le kilomètre. Alors que les mécanismes physiques initiateurs permettraient de regrouper

\author{
Différentes formes de dunes \\ selon l'alimentation et le régime \\ des vents
}


par exemple, les rides aquatiques, les dunes éoliennes et les dunes géantes martiennes qui ont toutes leur origine dans l'interaction entre le relief et le transport de sédiment.

Ces dix à vingt dernières années la communauté des physiciens et géophysiciens est très active et des résultats importants ont été publiés sur des aspects qui concernent particulièrement le sable ou de façon plus générale, les milieux granulaires. C'est ainsi que les différents aspects du mécanisme de la formation des dunes, et en conséquence la sélection de leur taille et de leur forme, sont aujourd'hui assez bien cernés (Andreotti et al. 2010, 2009). Ceci dit, le domaine reste très actif et très riche avec la nécessité de mener de nouveaux travaux pour répondre à des questions encore ouvertes, telles que la compréhension des rides éoliennes et la quantification du transport éolien dans des conditions naturelles (instationnarité, cohésion des grains...).

\section{Transport du sable et migration des dunes}

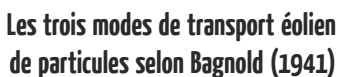

Suspension pour les petites particules (de diamètre moyen inférieur à quelques dizaines de micromètres) saltation pour les grains de sable entre 80 micromètres et un millimètre, reptation pour les particules plus grosses.
Le travail du major R. A. Bagnold, qui reste encore la référence dans le domaine, a permis de décomposer en trois modes le transport de sable par le vent au niveau du grain (schéma infra).

- la suspension pour les particules les plus petites $\left(D_{p}<80 \mu \mathrm{m}\right)$ transportées sur de longues distances par l'influence des structures turbulentes de l'écoulement. La quantité massique de particules mise en mouvement par suspension est quasi négligeable.

— la saltation pour les particules de taille moyenne qui font de petits bonds successifs $(80<$ $D_{p}<1000 \mu \mathrm{m}$ ). Le transport par saltation se fait sous la forme d'un nuage de particules évoluant à une hauteur de quelques centimètres au-dessus du sol.

- la reptation pour les plus grosses particules qui roulent sur le sol. Le déplacement par reptation est intimement lié à la saltation car ces gros grains entrent en mouvement par les impacts des grains de saltation sur le sol.

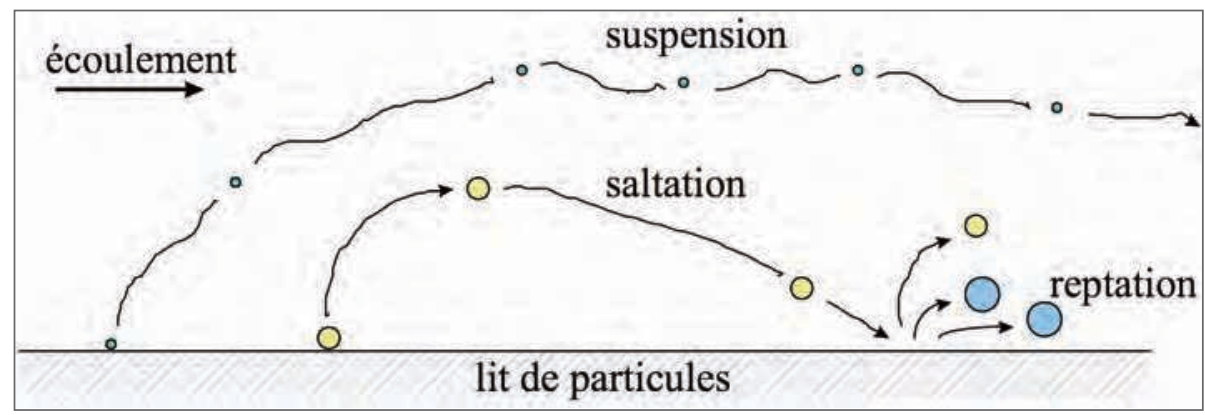

La recherche s'est penchée plus particulièrement sur la saltation qui est le mode de transport assurant une part prépondérante du flux massique de sable déplacé par le vent. Le flux massique est alors défini comme étant la masse de sable transportée par seconde et par mètre.

En y regardant de près, on constate que sur la face au vent d'une dune les particules se déplacent par saltation; elles s'accumulent au niveau de la crête de la dune. Une fois 
que la pente de la face abritée dépasse un certain angle d'équilibre, les particules tombent sous forme d'avalanches. Ce dernier phénomène d'avalanche permet à la dune de se déplacer en masse. Ces avalanches de grains de sable secs peuvent s'accompagner de l'émission d'un son grave et puissant : "Chant des dunes » (Andreotti et al. 2011). Les explications physiques de ce phénomène restent encore controversées, mais semblent le lier à des résonances impliquant les chocs entre les grains de sable. Ce chant des dunes et sa perception par les habitants locaux ont été décrits dans plusieurs récits d'explorateurs ou de romanciers allant de Marco Polo à Maupassant ou Darwin. Majoritairement, ces écrivains rapportent que les habitants font souvent une liaison entre ces bruits et des manifestations d'esprits, des voix de démons ou des miracles de saints.

Si l'on étudie un champ de barkhanes espacées, le flux de saltation est le flux interdune que l'on mesurerait entre les dunes. Une étude récente (Ould Ahmedou et al. 2007) a permis de quantifier ces différents flux dans un champ de barkhanes en Mauritanie. Elle montre en particulier que le flux dunaire (lié au déplacement de la dune en masse) est très faible comparé au flux interdunaire lié à la saltation.

Une conséquence de ce transport de sable et du déplacement des dunes de sable est l'ensablement des habitations, voire des villes (Chinguetti, par exemple), des infrastructures (routes, voies ferroviaires) et des terres cultivables (comme dans les oasis). Les habitants ont toujours lutté contre ces désagréments, le nomade se protège d'ailleurs par son chèche quand il est dehors. Dans sa tente, il utilise des nattes et des tissus pour fermer le tour de l'habitation. Quant aux sédentaires, ils utilisent des haies pour protéger les habitations et les cultures.

De façon générale, la lutte contre l'ensablement peut faire intervenir des méthodes d'ordre physique ou biologique (Mainguet 1995). Les méthodes physiques peuvent viser à dévier le sable pour éviter qu'il envahisse une zone donnée, à bloquer le sable à la source ou lors de son transport. Les méthodes biologiques visent essentiellement la fixation et la stabilisation par le reboisement des dunes ou des zones meubles.

On comprend bien que l'efficacité de ces systèmes restera limitée tant que les phénomènes de transport de sable et de la formation des structures éoliennes qui se forment selon les régimes d'érosion/déposition ne seront pas mieux compris. En effet, une meilleure compréhension physique de ces problématiques permettra, non seulement leur modélisation, mais aussi la simulation et le test de l'efficacité des moyens proposés pour la lutte contre l'ensablement.

Ceci dit, la lutte contre la désertification en général et les problèmes d'ensablement en particulier est un problème multidisciplinaire par excellence (Ould el-Moctar 2001). Du point de vue scientifique, il doit associer obligatoirement les physiciens, les mécaniciens, les géologues, les botanistes et surtout les sociologues. En effet, l'efficacité des apports techniques restera très limitée sans une approche humaine associée.

\section{Dune mouvante - Dune vivante}

A priori, le désert et en particulier les dunes de sable présentent des caractéristiques d'aridité extrême qui en font des endroits où l'on a du mal à imaginer la possibilité de la vie. Il suffit 
de visiter une dune de sable tôt le matin pour avoir la preuve du contraire et découvrir les traces d'êtres vivants qui témoignent d'une intense activité sur et dans la dune.

\section{Vie nocturne au Sahara \\ Cliché Mohamed Talbi. \\ Photographe environnemental. \\ IFPO : 15916}

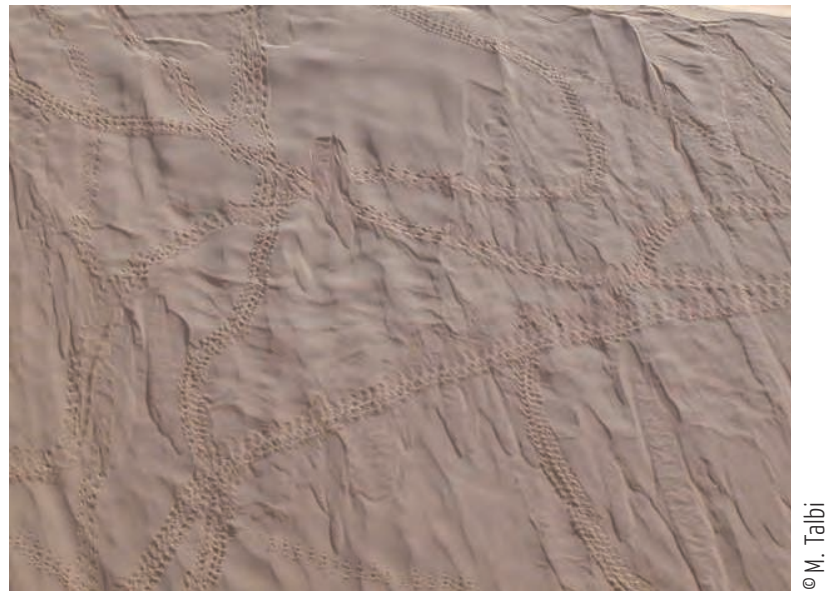

Une analyse minutieuse permet de révéler une importante activité microbienne. Des études de la diversité de ces micro-organismes et des conditions requises pour leur survie sur le sable existent dans la littérature (Gommeaux et al. 2005). On peut voir à titre d'exemple, ci-dessous, le résultat de visualisations par fluorescence effectuées sur des grains de sable prélevés en Mauritanie et au Qatar. On découvre pratiquement sur chaque grain de sable une colonie de micro-organismes vivants ou morts (Louge et al. 2013).

L'étude de ces bactéries et la maîtrise de leur culture éventuelle représentent une voie possible pour la stabilisation des dunes et donc la protection d'infrastructures contre le phénomène de l'ensablement.

\footnotetext{
Microbes sur un grain de sable

Les points orange sont des bactéries mortes, les points verts lumineux sont des bactéries vivantes. Photo de Sara Abdul-Majid, Anthony Hay et Michel Louge, 2012 (Qatar) (Louge et al. 2013)
}

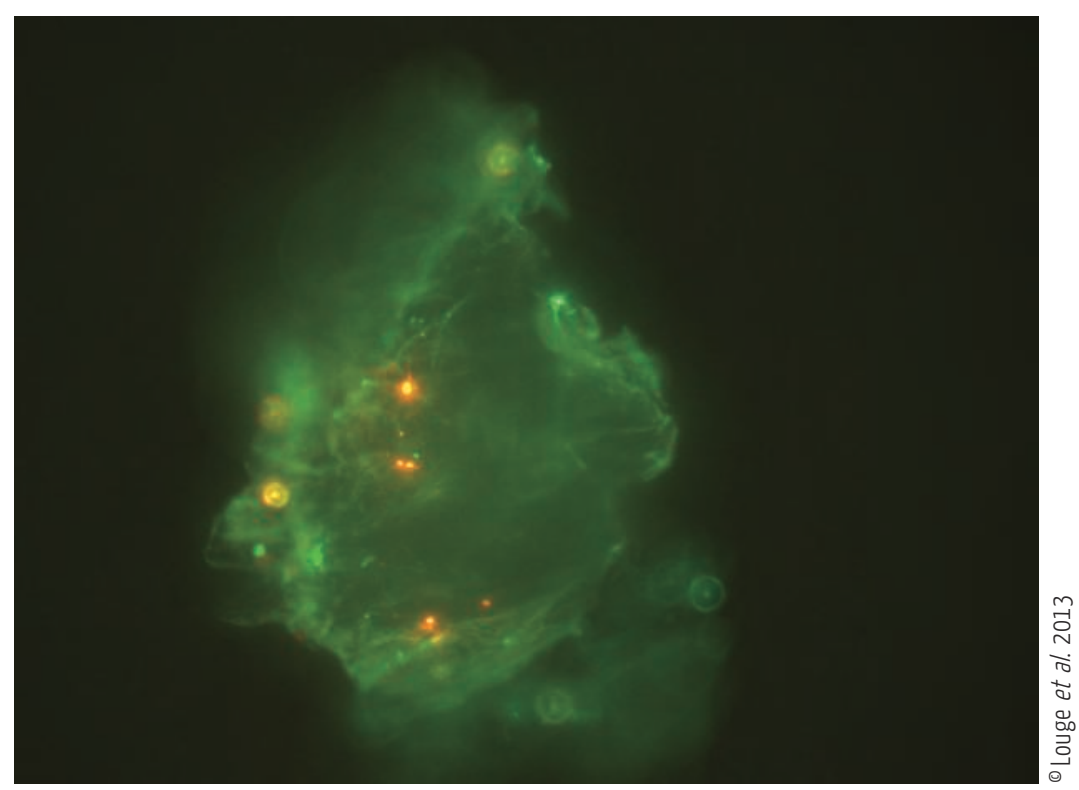




\section{Dunes extraterrestres}

Le programme d'exploration planétaire de la NASA a été l'occasion de plusieurs découvertes scientifiques concernant le voisinage de notre planète. Certaines ont soulevé des questions relatives aux phénomènes terrestres et ont ainsi contribué à améliorer la connaissance de notre propre planète. En effet, ces études relatives à Mars ou Vénus sont l'occasion de tester les hypothèses considérées dans les théories terrestres sous des conditions telles que la gravité et la pression atmosphérique où la température de surface sont différentes. Des scientifiques ont ainsi observé et étudié les tempêtes de sable et les dunes sur Mars et sur Vénus (ci-contre).

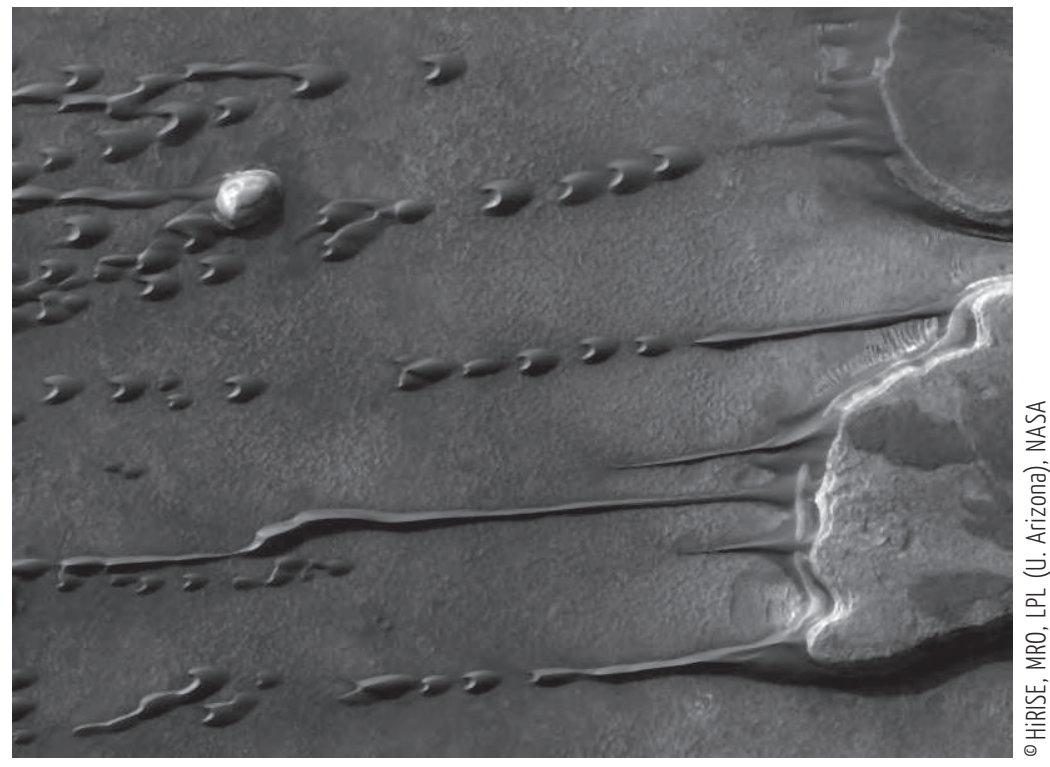

Ils ont parfois reproduit les conditions en soufflerie pour pouvoir prévoir la force de vent qui serait suffisante pour soulever les grains de sable sur Mars ou Vénus (Iversen, White 1982). De cette manière, on a pu noter que la vitesse équivalente pour soulever une particule, telle qu'un grain de sable, serait sur Mars dix fois plus élevée que celle nécessaire sur Terre. De plus les vents conséquents sont moins fréquents sur Mars. La présence d'édifices dunaires sur Mars s'expliquerait alors par l'atmosphère légère et la faible gravité de Mars qui permettent aux particules de voyager plus loin et plus vite. Effectivement, la vitesse indispensable au maintien de l'envol de la particule est dix fois plus faible que celle qu'il a fallu pour la soulever. Une revue des travaux concernant ces zones arides extraterrestres a été réalisée par Wells et al (1997). Si les structures et les processus éoliens sur Mars présentent des similitudes avec ceux sur Terre, la nature et la composition exacte du sédiment sur Mars (poussière, sable) restent à bien déterminer. Des travaux plus récents établissent des relations entre les dunes et les longueurs caractéristiques sur Terre, sur Mars et sur Vénus (Claudin, Andreotti 2006). Il s’agit là aussi d'un domaine en pleine expansion qui profite de la qualité des images satellitaires de haute définition.

Ceci illustre la force et l'intérêt de la démarche physique qui permet de définir des lois de comportement communes à ces milieux granulaires et à leur transport éolien. Cette analogie recouvre aussi la neige qui peut se trouver sous forme de grains transportés par le vent. À ce titre des analogies peuvent être trouvées avec certains des phénomènes (avalanches) ou des comportements rhéologiques que nous avons évoqués ici. Cependant la neige présente la spécificité du changement d'état (solide-liquide) ce qui complique encore l'exercice en raison de la taille des particules qui peut changer au cours du temps, ceci outre la présence de forces de cohésion importantes (Rognon 2006).

Ces approches physiques ont permis des avancées, mais beaucoup de points demeurent encore à élucider. Il nous semble entre autres que ces matières naturelles et non transformées gagneraient à être davantage abordées par l'anthropologie des techniques. 


\section{NOTES}

Photo d'ouverture: Vue d'une barkhane avec un réseau de petites rides éoliennes sur la dune, des mégarides en dessous de la dune et une avalanche qui vient de se déclencher. (Cl. A. Valance, 2004, Mauritanie).

\section{REMERCIEMENTS}

Je tiens à remercier Alexandre Valance, Pascal Dupont et Michel Louge qui m’ont initié à ce domaine, guidant mes pas sur le chemin de la découverte de mes dunes natales.

\section{RÉFÉRENCES}

Andreotti, B., Forterre, Y., et Pouliquen, O. 2011 Les Milieux granulaires, entre fluide et solide. Les Ulis : EDP Sciences.

Andreotti, B., Claudin, P., Pouliquen, O. 2010 Measurements of the aeolian sand transport saturation length, Geomorphology 123 (2010) : 343-348.

Andreotti, B., Fourrière, A., Ould-Kaddour, F. E al. 2009 Giant aeolian dune size determined by the average depth of the atmospheric boundary layer, Nature 457 : 1120-1123.

Bagnold, R. A. 1941 The Physics of blown-sand and desert dunes, New-York : Morrow.

Claudin, P., Andreotti, B. 2006 A scaling law for aeolian dunes on Mars, Venus, Earth, and for subaqueous ripples. Earth Planet. Sci. Lett. 252 : 30-44.

Claudin, P. 1999 La Physique des tas de sables : description phénoménologique de la propagation des contraintes dans les matériaux granulaires. Université Paris XI (thèse de Doctorat).

Gommeaux, M. 2005 Bactéries du sable de Merzouga. Université Aix-Marseille III (thèse de Doctorat).

Louge, M. Y., Valance, A., Ould el-Moctar, A. E al. 2013 Temperature and humidity within a mobile barchan sand dune, implications for microbial survival, J. Geophys. Res. Earth Surf., 118, doi:10.1002/2013JF002839 consulté le 16 novembre 2014.

Iversen, J. D., White, B. R. 1982 Saltation threshold on Earth, Mars and Venus, Sedimentology 29 : $111-119$.

Mainguet, M. 1995 L'Homme et la sécheresse. Paris : Masson.

Ould Ahmedou, D., Ould Mahfoud, A., Dupont, P., Ould el-Moctar, A. E al. 2007 Barkhane dune mobility in Mauritania related to dune and inter-dune sand fluxes, J. Geophys. Res., 112, F02016, doi:10.1029/2006JF000500 consulté le 16 novembre 2014.

Ould el-Moctar, A. (ed.) 2001 Formation et Migration des Dunes. 2e Atelier International de Nouakchott.

Rognon, P. 2006 Rhéologie des matériaux granulaires cohésifs. Application aux avalanches de neige dense. Paris : École nationale des Ponts et Chaussées (thèse).

Wells, G. L., Zimbelman, J. R. 1997 Extra terrestrial arid surface processes. In Arid Zone Geomorphology, $2^{\text {nd }}$ ed., D.S.G. Thomas (ed.). Wiley : Cichester : 659-690.

\section{POUR CITER CET ARTICLE}

Ould el-Moctar, A. 2014 Le Sable du point de vue de la physique, in S. Boulay \& M.-L. Gélard, Vivre le sable! Corps, matière et sociétés, Techniques \& Culture 61 : 28-41. 


\section{RÉSUMÉ}

Le Sable du point de vue de la physique. Le sable joue un rôle économique, environnemental et culturel important dans la vie des habitants des zones désertiques. Cette matière s'intégrant parfois dans la composition de matériaux utiles, à la construction par exemple, peut présenter par son mouvement ou son accumulation des désagréments, voire des dangers importants. Cela se traduit par une gêne personnelle lors des tempêtes de sable ou par des problèmes d'ensablement plus globaux et plus contraignants du point de vue du développement de ces régions. La compréhension des caractéristiques du sable et des phénomènes liés à son déplacement deviennent alors un enjeu important. C'est une matière qui peut fasciner par différents aspects, notamment le comportement physique qu'elle a en commun avec les autres matériaux granulaires. Le sable peut en effet se comporter, selon les situations, comme s'il était un gaz, un liquide ou un solide. Dans un tas de sable, les contraintes s'organisent selon des réseaux particuliers présentant des formes de voûte. Cette répartition des contraintes est à l'origine de plusieurs comportements non intuitifs qui caractérisent ces milieux. En fonction des circonstances (disponibilité du sable, conditions de vent, granulométrie...), le sable s'organise sous forme d'édifices (rides, dunes, champ dunaire...) présentant des longueurs caractéristiques différentes. Des structures similaires sont observables sous les rivières ou les océans et sur d'autres planètes, telles que Mars ou Vénus. Comprendre les phénomènes physiques mis en jeu dans la formation de ces structures et dans le transport éolien du sable représente une étape importante dans une démarche multidisciplinaire visant à trouver des solutions pour lutter efficacement contre les problèmes d'ensablement et de désertification.

\section{ABSTRACT}

Sand: physics' perspectives. Sand plays an important economic, environmental and cultural role in the lives of people in desert areas. This material which is sometimes integrated into useful materials like for construction, for example, may cause some of annoyances and even significant hazards by its accumulation or displacement. This may result in personal gene during sandstorms or global problems of desertification which are more disturbing in terms of the development of these regions. Understanding the physical behavior of sand and basic phenomena related to its transport becomes an important issue. Sand has various fascinating aspects including physical behavior it has in common with other granular matter. Thus the sand can behave in different situations as if it were a gas, a liquid or a solid. In a pile of sand, the constraints are organized in networks with specific forms of arch. This stress distribution is at the origin of several non-intuitive behaviors that characterize these media. Depending on the circumstances (availability of sand, wind conditions, granulometry, ...), the sand is organized in the form of various geometrical shapes (ripples, dunes, dune field, ...) with different characteristic lengths. Similar structures can be observed under rivers or oceans or on other planets such as Mars or Venus. Understanding the physical phenomena involved in the formation of these structures and aeolian transport of sand is an important step in a multidisciplinary approach aimed at finding effective solutions to the desertification problems.

\section{MOTS CLÉS}

Sable, dune, désert, physique du sable, transport éolien, flux de sable

\section{KEYWORDS}

Sand, dune, desert, sand physics, aeolian transport, sand flux 